\title{
Multiband superconductivity and nanoscale inhomogeneity at oxide interfaces
}

\author{
S. Caprara, ${ }^{1,2}$ J. Biscaras, ${ }^{3}$ N. Bergeal,,${ }^{3}$ D. Bucheli, ${ }^{1}$ S. Hurand,${ }^{3}$ C. Feuillet-Palma, ${ }^{3}$ A. Rastogi,${ }^{4}$ R. C. Budhani, ${ }^{4,5}$ \\ J. Lesueur, ${ }^{3}$ and M. Grilli ${ }^{1,2}$ \\ ${ }^{1}$ Dipartimento di Fisica, Università di Roma "La Sapienza", Piazzale Aldo Moro 5, 00185 Roma, Italy \\ ${ }^{2}$ ISC-CNR and Consorzio Nazionale Interuniversitario per le Scienze Fisiche della Materia, Unità di Roma "Sapienza”, Roma, Italy \\ ${ }^{3}$ LPEM-UMR8213/CNRS-ESPCI Paris Tech, UPMC, 10 rue Vauquelin, 75005 Paris, France \\ ${ }^{4}$ Department of Physics, Condensed Matter - Low Dimensional Systems Laboratory, \\ Indian Institute of Technology Kanpur, Kanpur, 208016, India \\ ${ }^{5}$ National Physical Laboratory, New Delhi, 110012, India
}

(Received 28 March 2013; published 22 July 2013)

\begin{abstract}
The two-dimensional electron gas at the $\mathrm{LaTiO}_{3} / \mathrm{SrTiO}_{3}$ or $\mathrm{LaAlO}_{3} / \mathrm{SrTiO}_{3}$ oxide interfaces becomes superconducting when the carrier density is tuned by gating. The measured resistance and superfluid density reveal an inhomogeneous superconductivity resulting from percolation of filamentary structures of superconducting "puddles" with randomly distributed critical temperatures, embedded in a nonsuperconducting matrix. Following the evidence that superconductivity is related to the appearance of high-mobility carriers, we model intrapuddle superconductivity by a multiband system within a weak coupling BCS scheme. The microscopic parameters, extracted by fitting the transport data with a percolative model, yield a consistent description of the dependence of the average intrapuddle critical temperature and superfluid density on the carrier density.
\end{abstract}

DOI: 10.1103/PhysRevB.88.020504

PACS number(s): 74.81.-g, 74.20.-z, 74.25.fc, 74.78.-w

The discovery of superconductivity in the two-dimensional electron gas (2DEG) formed at certain oxide interfaces such as $\mathrm{LaAlO}_{3} / \mathrm{SrTiO}_{3}\left(\mathrm{LAO} / \mathrm{STO}\right.$ ) or $\mathrm{LaTiO}_{3} / \mathrm{SrTiO}_{3}(\mathrm{LTO} / \mathrm{STO})^{1-4}$ has attracted great interest and stimulated intense research. The possibility of tuning the carrier density by means of electrostatic gating in these extremely two-dimensional superconductors (the Fermi wavelength being smaller than the thickness of the gas ${ }^{4}$ ) opens new opportunities to study fundamental issues in quantum fluids. ${ }^{5}$ These 2DEGs are rich quantum matter systems displaying magnetism ${ }^{6-11}$ and strong tunable spinorbit coupling. ${ }^{12,13}$ Recent magnetotransport measurements revealed the existence of two kinds of carriers in LTO/STO, with high and low mobility respectively. ${ }^{4}$ It has been suggested that superconductivity is related to the appearance of the high-mobility carriers ${ }^{4,14}$ with an inhomogeneous character, i.e., superconducting "puddles" embedded in a (weakly localizing) metallic background. ${ }^{5}$ Direct superfluid density ${ }^{11}$ and magnetic ${ }^{7}$ measurements detect strong inhomogeneity at micrometric scales. Recent current distribution ${ }^{15}$ and surface potential studies ${ }^{16}$ reveal striped structures at these interfaces. Given all these evidences for inhomogeneities, one has first to analyze the role of disorder. Our aim is, on the one hand, to infer a more detailed structure of the inhomogeneous state and, on the other hand, to provide a description for superconductivity in an inhomogeneous system with different kinds of carriers. We first show that resistance measurements ${ }^{4}$ and the topographic mapping of the superfluid density at micrometric scale ${ }^{11}$ can be phenomenologically accounted for within a scheme of a percolative inhomogeneous 2DEG with poor long-distance connectivity, but substantial superconducting (SC) current loops to warrant a high fraction of diamagnetic response. We then show that the properties of the SC puddles (e.g., their fraction, and critical temperatures) can be extracted from experiments and used within a microscopic model for intrapuddle multicarrier pairing, gaining insight about the pairing mechanism. Although some features of the diamagnetic response are seemingly related to strong SC coupling, ${ }^{11}$ we show that inhomogeneities and multiband superconductivity fully account for the behavior of these systems within a standard weak coupling BCS scheme.

Sheet resistance. We have recently shown that the percolative SC transition in inhomogeneous systems, such as the oxide interfaces, is well described by the effective medium theory (EMT). ${ }^{17}$ For samples exhibiting a resistance drop due to superconductivity, the measured sheet resistance of LTO/STO is well fitted by the EMT with a SC fraction $w<1$ (the SC puddles). Each puddle has a random local critical temperature $T_{c}$, which we assume to have a Gaussian distribution, with mean $\bar{T}_{c}$ and width $\gamma$. The weight $w$ of the $T_{c}$ distribution represents the fraction of SC puddles and the non-SC fraction $1-w$ represents the metallic background (see Ref. 17 for details). The sheet resistance at temperature $T$ is

$$
R(T)=R^{\infty}\left[w \operatorname{erf}\left(\frac{T-\bar{T}_{c}}{\sqrt{2} \gamma}\right)+1-w\right],
$$

with fitting parameters $R^{\infty}$ (high-temperature sheet resistance), $w, \bar{T}_{c}$, and $\gamma$. The percolative SC transition, marked by a vanishing $R$, takes place at a temperature $T_{p} \leqslant \bar{T}_{c}$, such that

$$
\operatorname{erf}\left(\frac{T_{p}-\bar{T}_{c}}{\sqrt{2} \gamma}\right)=1-\frac{1}{w}
$$

This equation has a solution for $T_{p}$ only if $w \geqslant \frac{1}{2}$, i.e., if the SC fraction can percolate in the two-dimensional system. When $T_{p}$ is negative or not defined, the system remains resistive down to $T=0$, although a significant reduction of $R(T)$ may still occur, witnessing the presence of a sizable (though not percolating) SC fraction.

Figure 1(a) reports the measured sheet resistance as a function of $T$ already reported in Ref. 4 for various values of the gate voltage $V_{g}$ in a LTO/STO sample, and the fitting 


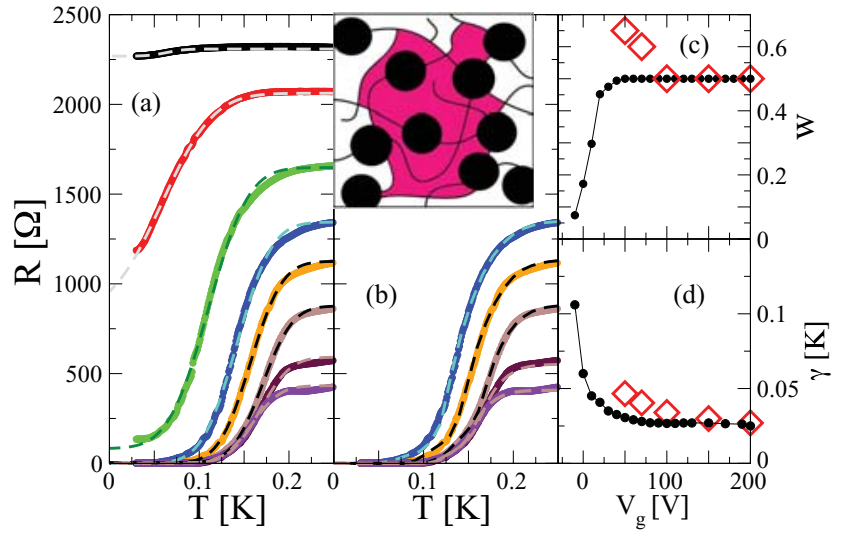

FIG. 1. (Color online) (a) Sheet resistance as a function of temperature in a LTO/STO sample for various values of the gate voltage. From top to bottom $V_{g}=-10,10,30,50,70,100,150$, 200 V. Thick lines are experimental data from Ref. 4, while the dashed lines are the EMT fits (see text). (b) From top to bottom $V_{g}=50,70,100,150,200 \mathrm{~V}$. Thick lines are experimental data from Ref. 4, while the dashed lines are the theoretical fits using the RRN (see text). Inset: sketch of an inhomogeneous RRN system with a SC filamentary + superpuddles cluster. The shaded region is a closed loop contributing to the diamagnetic response. (c) SC fraction $w$ extracted from the fits of the sheet resistance within EMT (filled circles, solid line) and within the RRN (open diamonds). (d) Width of the Gaussian distribution of $T_{c}$ used to fit the sheet resistance within the EMT (filled circles, solid line) and within the RRN (open diamonds).

EMT curves. $V_{g}$ changes the electron density at the interface thereby changing the distribution of the SC regions. From the fits we extract the average intrapuddle critical temperature $\bar{T}_{c}$ [corresponding to the temperature of maximal slope of $R(T)$ within EMT], which is reported in Fig. 3(a) as a function of $V_{g}$ (red solid line and open circles). The fits also provide the fraction of SC regions $w$ [displayed in Fig. 1(c) as a function of $V_{g}$ ]. Since EMT disregards spatial correlations, the simultaneous request of a percolating SC cluster and the tailish shape of the resistance near percolation forces the fraction of SC puddles to be $w \approx \frac{1}{2}$ [Fig. 1(c), see also Refs. 17 and 18]. Figure $1(\mathrm{~d})$ reports the width $\gamma$ of the Gaussian distribution of $T_{c}$, which strongly increases as the fraction $w$ of the SC puddles goes to zero. This is rather natural because the density decrease emphasizes the effects of disorder so that fluctuations of the local superconductivity increase, leading to a substantial broadening of the $T_{c}$ distribution. We will give later a more precise description of this phenomenon within the two-band model.

Superfluid density. The superfluid density $J_{S}$ was measured in LAO/STO interfaces using a scanning SQUID probe averaging over micrometric regions. ${ }^{11}$ This technique is not sensitive to submicrometric inhomogeneities, but revealed a distribution of $J_{s}$ on the micrometric scale within a given sample, thereby supporting the idea of inhomogeneous $2 \mathrm{DEG}$ at these interfaces. Since the sheet resistance curves of these systems are similar to those of LTO/STO interfaces, we assume that our percolative EMT analysis also applies in this case and that the measured local $J_{s}$ is an average over an inhomogeneous state of submicrometric puddles. ${ }^{19}$
To capture this effect, we extend the EMT to small but finite frequency $\omega$. We model the metallic regions with a Drude-like complex conductivity $\sigma_{N}(\omega)=B /(A+i \omega)$ and the SC regions with a purely reactive conductivity $\sigma_{S}(\omega)=$ $B /(i \omega)$. We define $\rho_{S}(\omega)=1 / \sigma_{S}(\omega)=i \omega / B$ and $\rho_{N}(\omega)=$ $1 / \sigma_{N}(\omega)=\rho_{0}+\rho_{S}(\omega)$, with $\rho_{0}=A / B$. At high temperature, the system is metallic and $\rho(\omega)=1 / \sigma(\omega)=\rho_{N}(\omega)$ everywhere. However, within the SC puddles, $\rho_{0}$ vanishes as soon as the local critical temperature is reached. The solution of the EMT equation, neglecting higher frequency terms $\sim \omega^{2}$, is

$$
\rho(\omega) \approx \rho_{0}\left(w_{N}-w_{S}\right) \vartheta\left(w_{N}-w_{S}\right)+\frac{\rho_{S}(\omega)}{\left|w_{N}-w_{S}\right|},
$$

where $\vartheta(x)$ is the Heaviside step function, $w_{S}$ is the fraction of puddles that have become SC (at a given temperature $T$ ), and $w_{N}=1-w_{S}$ is the metallic fraction (resulting both from puddles that have not yet become SC and from the metallic background). Evidently, when $w_{N}>w_{S}$, the system displays a Drude-like complex conductivity. However, below the percolation temperature $T_{p}$ (if any), $w_{S}>w_{N}$, and we find a purely reactive conductivity

$$
\sigma(\omega)=\frac{1}{\rho(\omega)}=\frac{A\left(w_{S}-w_{N}\right)}{i \omega} .
$$

Therefore, for $T<T_{p}$ and $w$ given by Eq. (1), the superfluid density of the percolating network is

$$
\begin{aligned}
J_{s} \propto w_{S}-w_{N} & =w-1-w \operatorname{erf}\left(\frac{T-\bar{T}_{c}}{\sqrt{2} \gamma}\right) \\
& =w\left[\operatorname{erf}\left(\frac{T_{p}-\bar{T}_{c}}{\sqrt{2} \gamma}\right)-\operatorname{erf}\left(\frac{T-\bar{T}_{c}}{\sqrt{2} \gamma}\right)\right] .
\end{aligned}
$$

Figure 2(a) reports this micrometrically averaged superfluid density together with our EMT fits [Eq. (2)] at various gate voltages $V_{g}$. Remarkably, the $T$ dependence of $J_{s}(T) / J_{S}(T=$ $0)$ mimics the qualitative behavior of the BCS prediction, but may quantitatively deviate from it. The slope at the transition, for instance, is ruled by the width $\gamma$ of the distribution of $T_{c}$.
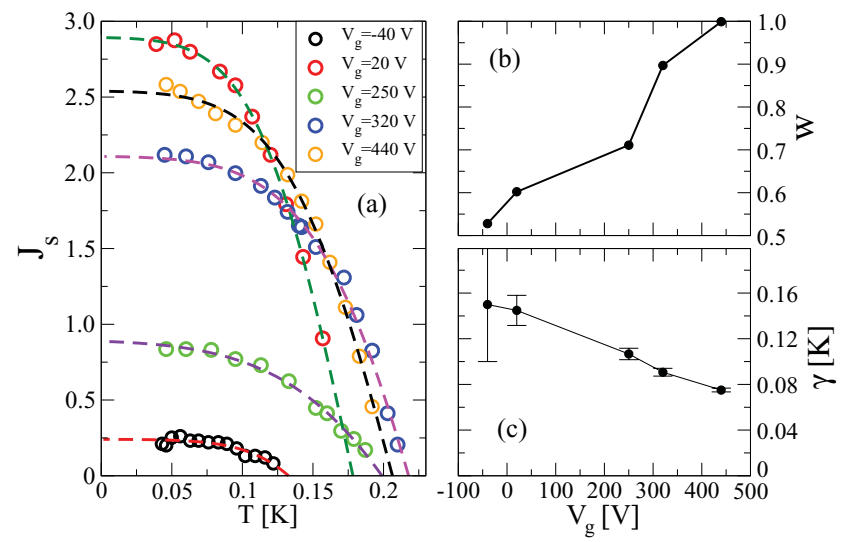

FIG. 2. (Color online) (a) EMT fits of the superfluid density in LAO/STO (lines) as a function of temperature for various gate voltages. The experimental data (symbols) are extracted from Ref. 11. (b) SC fraction $w$ responsible for the diamagnetic response. (c) Width of the Gaussian distribution of $T_{c}$ used to fit $J_{s}$. 
We can thus account for the deviations from standard BCS observed in Ref. 11, which were attributed to a tendency to strong coupling. Our alternative explanation for these deviations is that the system is in a weak coupling regime (see below), but the percolative superfluid density is ruled by the submicrometric distribution of inhomogeneities averaged at the micron scale by the SQUID pickup loop used in Ref. 11. Although not directly measured, the typical size of SC inhomogeneities has been estimated by scaling arguments to be in the $100 \mathrm{~nm}$ range..$^{5}$ Moreover the properties of channels down to $500 \mathrm{~nm}$ wide are similar to the large-scale materials. ${ }^{20}$ Therefore a mean-field approach is fully justified here. The fits yield the fraction $w$ of SC puddles extracted from diamagnetic measurements which is reported in Fig. 2(b). It ranges from $\frac{1}{2}$ to 1 , and is therefore always larger than the fraction obtained from transport measurements. This is fully consistent within a percolative description of the system: resistance measurements mainly probe the direct percolative path, regardless of dead ends and disconnected SC regions, ${ }^{21}$ while screening measurements are sensitive to all SC loops, even when not connected to the backbone. In this case, the diamagnetic fraction can be nearly one, while the connectivity for macroscopic transport can be very small (or even vanishing) if many SC puddles or loops are disconnected [see the sketch in the inset of Fig. 1(b)]. Figure 2(c) displays the behavior of the width $\gamma$ of the $T_{c}$ distribution. Despite the differences in material and physical quantities, $\gamma$ has a similar behavior to the $\gamma$ from transport in LTO/STO [Fig. 1(d)] and is of comparable magnitude.

Of course, a complete proof of these arguments would require a model where space correlations, distinguishing closed loops (relevant for diamagnetism) and connected paths (relevant for transport), are considered. While a RRN model for transport has been found (see below), a similar model to describe the diamagnetic response is presently not available. However, our scenario could be experimentally tested by detecting different diamagnetic responses in field-cooled and zero-field-cooled samples. In the first case one expects substantially smaller diamagnetic fraction, since a substantial flux would be trapped in the normal part encircled by the SC loops [see the shaded region in the sketch inset of Fig. 2(b)].

Effect of space correlations. The explanation of the above discrepancy between the SC weight $w$ in transport and magnetic experiments rests on the filamentary structure of the $\mathrm{SC}$ fraction at the oxide interfaces, which is one of the two main outcomes of our work. However, the above results (and fits) have been obtained within the EMT, which is a meanfield-like approach neglecting space correlations. Therefore, to substantiate the above interpretation, it is important to test how robust is the finding that the SC fraction involved in transport is rather small, $w \approx \frac{1}{2}$, within a model, which takes into account the space correlations inherent to the filamentary structure. To this purpose, we solve a random resistor network (RRN) where the SC regions only occur on a cluster with strong space correlations embedded in a metallic matrix. Based on a preliminary analysis, ${ }^{22}$ showing that the tailish sheet resistance implies both a dense SC cluster at short distances and a filamentary structure at larger distance, we numerically generate a fractal-like structure. ${ }^{22,23}$ Due to the poor long-distance connectivity, percolation only occurs when the resistance of almost all bonds has been switched off. For a Gaussian distribution of critical temperatures, the system is thus forced to explore the low-temperature asymptotic of the distribution, thereby producing a tailish resistance. ${ }^{24}$ On the other hand, a purely filamentary structure, although dense at short distance due to the fractal-like cluster, is still too faint $(w \lesssim 0.3)$ and fails to reproduce accurately the hightemperature part of the $R(T)$ curves. Thus, in order to vary the density of the SC cluster without crucially changing the long-range connectivity, we decorate the filamentary tree with randomly distributed circular broader superconducting regions [see inset of Fig. 1(b)], henceforth called "superpuddles", their number being chosen to yield RRNs with weights $w$ ranging from 0.3 to $0.7 .^{25}$ Figure 1(b) reports fits of the sheet resistance using resistivity curves calculated on such trees. Noticeably, even though the $\mathrm{SC}$ weight $w$ is not forced to $\frac{1}{2}$ like in EMT, we still find that the sheet resistance only acquires a tailish behavior if $0.5 \lesssim w \lesssim 0.65$ [diamonds in Fig. 1(c)], where the lower bound is imposed by the high slope at intermediate temperatures and the upper bound is due to the tailish behavior close to percolation. Figure 1(d) reports the width $\gamma$ of the Gaussian distribution of $T_{c}$ obtained within the tree model (diamonds). One can see that $\gamma$ follows the same qualitative behavior as in the EMT case with a substantial increase upon lowering the voltage. As a conclusion, EMT and RRN models lead to very close results about the $T_{c}$ distribution and its variation with the gate voltage provided a filamentary tree structure is used for the RRN.

Multiband BCS scenario. The inhomogeneous character of the oxide interfaces, clearly apparent both in the normal-state $[R(T)]$ and in the SC $\left[J_{S}(T)\right]$ properties, entails a distribution of local critical temperatures with an average depending on the overall density (i.e., gating) $\bar{T}_{c}\left(V_{g}\right)$. This dependence can be extracted from the resistance fits (Fig. 3) and provides insight on the intrapuddle pairing mechanism. A recent magnetotransport analysis ${ }^{4}$ demonstrated the coexistence of a rather large density of low-mobility carriers (LMCs) with a smaller density of high-mobility carriers (HMCs) in the SC regime of LTO/STO. In particular, superconductivity seems to

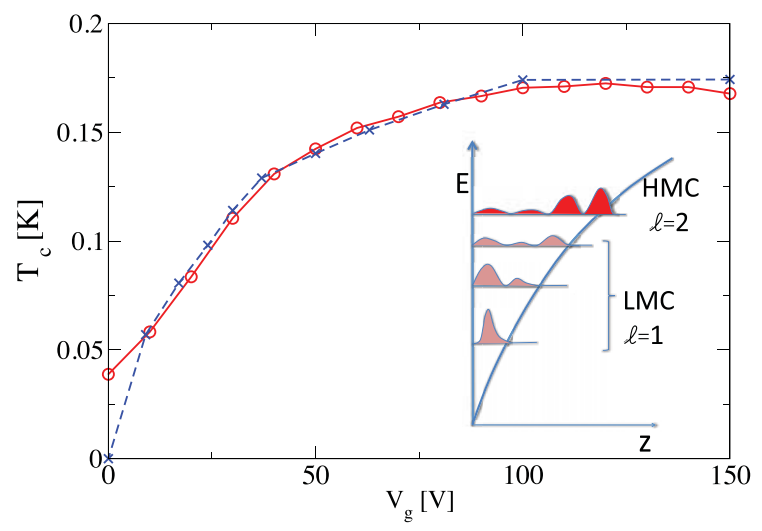

FIG. 3. (Color online) $\bar{T}_{c}$ extracted from the EMT fit to experimental data (red solid line and open circles) and the theoretical $T_{c}$ calculated within the two-band model (see text), as a function of the gate voltage $V_{g}$ (blue dashed line and crosses). Inset: schematic sub-band structure of the $2 \mathrm{DEG}$ arising from the confining potential at the interface. 
be triggered by the presence of the HMC. A similar behavior seems to occur in $\mathrm{LAO} / \mathrm{STO}{ }^{26}$ We recall that the $2 \mathrm{DEG}$ formed at the oxide interface may be described as a multiband system, ${ }^{4,27-31}$ arising from the electronic confinement in the $z$ direction perpendicular to the interface (see inset in Fig. 3). Thus the LMCs occupy lower states in the multilevel structure and their more marked two-dimensional character accounts for their lower mobility. On the other hand the carriers occupying the higher sub-bands are less confined along $z$ and more mobile. According to this scenario the occurrence of superconductivity may be captured by means of a multiband BCS-like Hamiltonian ${ }^{18}$ : We represent the whole set of lowlying bands with one single sub-band $\left[\varepsilon_{\ell}\left(k_{x}, k_{y},\right)\right.$ with $\left.\ell=1\right]$ collecting all the LMC with vanishingly small $T_{c}$, while the occupation by HMC of the upper sub-band(s) $\varepsilon_{\ell}\left(k_{x}, k_{y},\right)$ with $\ell=2$ gives rise to a finite $T_{c}$. This scenario can now be related to the inhomogeneous structure of the system on the $x y$ interface by assuming that the $\mathrm{SC}$ puddles are regions where the sub-band $\ell=2$ is locally filled, whereas the (weakly localized) metallic background corresponds to regions where the sub-band $\ell=2$ is empty.

To reproduce the experimental results, we are led to a suitable choice of the pairing amplitudes $g_{\ell \ell^{\prime}}$ (intraband for $\ell=\ell^{\prime}$, interband, for $\left.\ell \neq \ell^{\prime}\right): g_{11} \ll\left(g_{12}, g_{21}\right) \ll g_{22}$ (this condition is consistent with the analysis of a two-band model in Ref. 32). According to the standard BCS approach, the pairing amplitudes are only effective in a window $\mid \varepsilon_{\ell}(\mathbf{k})-$ $\mu|,| \varepsilon_{\ell^{\prime}}\left(\mathbf{k}^{\prime}\right)-\mu \mid \leqslant \hbar \omega_{0}$, where $\omega_{0}$ is a characteristic frequency of the pairing mediator and $\mu$ is the chemical potential. We assume that the bottoms of the two sub-bands are well separated, $\bar{\varepsilon}_{2}-\bar{\varepsilon}_{1} \gg \hbar \omega_{0}$, and take $\bar{\varepsilon}_{2}=0$ as the reference energy level.

While more general (though standard) expressions are derived in the Supplemental Material, ${ }^{18}$ here we only report the expressions obtained for $g_{11}=g_{12}=g_{21}=0$, so that the system is not SC until the carrier density reaches the value such that $\mu=0$. For $0<\mu<\hbar \omega_{0}, T_{c}$ increases with increasing $\mu$

$$
T_{c} \approx 1.14 \sqrt{\hbar \omega_{0} \mu} \mathrm{e}^{-1 / \lambda_{2}},
$$

where we merged into the single dimensionless coupling $\lambda_{2} \equiv N_{2}^{0} g_{22}$ the intraband $g_{22}$ coupling and the density of states $N_{2}^{0}$ of $\varepsilon_{2}(\mathbf{k})$. For $\mu>\hbar \omega_{0}, T_{c}$ saturates to its maximum (BCS) value $T_{c}^{\max } \approx 1.14 \hbar \omega_{0} \mathrm{e}^{-1 / \lambda_{2}}$. Thus, $T_{c}(\mu) \simeq 0$, for $\mu<0$, $T_{c}(\mu)=T_{c}^{\max } \sqrt{\mu / \hbar \omega_{0}}$, for $0 \leqslant \mu \leqslant \hbar \omega_{0}, T_{c}(\mu)=T_{c}^{\max }$, for $\mu \geqslant \hbar \omega_{0}$, and the range of variation of $\mu$, which corresponds to an increasing $T_{c}$ is a direct measure of the characteristic energy scale of the pairing mediator, $\hbar \omega_{0}$.

We now proceed to fit the curve $\bar{T}_{c}\left(V_{g}\right)$ extracted from the experimental data by means of EMT, with our theoretical curve $T_{c}(\mu)$. The relation between $V_{g}$ and $\mu$ is only approximately linear and its determination is described in detail in the Supplemental Material. ${ }^{18}$ The resulting critical temperature for various values of $V_{g}$ is reported in Fig. 3 (blue dashed line and crosses). From the fit, we finally obtain the dimensionless coupling constant $\lambda_{2} \approx 0.25$ (which, consistently with our assumption, is in the weak coupling regime) and $\hbar \omega_{0} \approx 23 \mathrm{meV}$, which is the typical energy of phonons in STO. ${ }^{33}$

In conclusion, we describe superconductivity in LAO/STO and LTO/STO oxide interfaces within a scenario in which SC puddles form a percolating network. In this framework, the sheet resistance of LTO/STO interfaces is very well described by EMT or by a tree model of RRN for an inhomogeneous 2DEG with a substantial filamentary character. Fitting the experiments, we are able to extract the random distribution of $T_{c}$ at various carrier densities (or $V_{g}$ ). A similar approach is also adopted to fit the micrometrically averaged superfluid density in LAO/STO. ${ }^{11}$ Assuming an effective two-band model with superconductivity triggered by the presence of few high-mobility carriers in a higher band, we account for the density dependence of the intrapuddle $T_{c}$ within a simple BCS weak coupling scheme. As an important byproduct, we find that the range of variation in $V_{g}$ of the (average) intrapuddle $T_{c}$ is directly related (via the chemical potential $\mu$ ) to the typical energy scale of the pairing mediator $\hbar \omega_{0}$, compatible with phonon-mediated superconductivity.

We acknowledge interesting discussions with L. Benfatto, C. Castellani, and C. Di Castro. S.C. and M.G. acknowledge financial support from "University Research Project" of the "Sapienza" University Grant No. C26A125JMB. This work has been supported by the Region Ile-de-France in the framework of CNano IdF and Sesame programs, and by the DGA Ph.D. program. Research in India was funded by the Department of Information Technology, Government of India.
${ }^{1}$ N. Reyren, S. Thiel, A. D. Caviglia, L. Fitting Kourkoutis, G. Hammerl, C. Richter, C. W. Schneider, T. Kopp, A.-S. Rüetschi, D. Jaccard, M. Gabay, D. A. Muller, J.-M. Triscone, and J. Mannhart, Science 317, 1196 (2007).

${ }^{2}$ A. D. Caviglia et al., Nature (London) 456, 624 (2008).

${ }^{3}$ J. Biscaras, N. Bergeal, A. Kushwaha, T. Wolf, A. Rastogi, R. C. Budhani, and J. Lesueur, Nature Commun. 1, 89 (2010).

${ }^{4}$ J. Biscaras, N. Bergeal, S. Hurand, C. Grossetete, A. Rastogi, R. C. Budhani, D. LeBoeuf, C. Proust, and J. Lesueur, Phys. Rev. Lett. 108, 247004 (2012).
${ }^{5}$ J. Biscaras, N. Bergeal, S. Hurand, C. Feuillet-Palma, A. Rastogi, R. C. Budhani, M. Grilli, S. Caprara, and J. Lesueur, Nature Mater. 12, 542 (2013).

${ }^{6}$ Ariando, X. Wang, G. Baskaran, Z. Q. Liu, J. Huijben, J. B. Yi, A. Annadi, A. Roy Barman, A. Rusydi, S. Dhar, Y. P. Feng, J. Ding, H. Hilgenkamp, and T. Venkatesan, Nature Commun. 2, 188 (2011).

${ }^{7}$ Lu Li, C. Richter, J. Mannhart, and R. C. Ashoori, Nature Phys. 7, 762 (2011).

${ }^{8}$ J. A. Bert, B. Kalisky, C. Bell, M. Kim, Y. Hikita, H. Y. Hwang, and K. A. Moler, Nature Phys. 7, 767 (2011). 
${ }^{9}$ D. A. Dikin, M. Mehta, C. W. Bark, C. M. Folkman, C. B. Eom, and V. Chandrasekhar, Phys. Rev. Lett. 107, 056802 (2011).

${ }^{10}$ M. M. Mehta, D. A. Dikin, C. W. Bark, S. Ryu, C. M. Folkman, C. B. Eom, and V. Chandrasekhar, Nature Commun. 3, 955 (2012).

${ }^{11}$ Julie A. Bert, Katja C. Nowack, Beena Kalisky, Hilary Noad, John R. Kirtley, Chris Bell, Hiroki K. Sato, Masayuki Hosoda, Yasayuki Hikita, Harold Y. Hwang, and Kathryn A. Moler, Phys. Rev. B 86, 060503(R) (2012).

${ }^{12}$ A. D. Caviglia, M. Gabay, S. Gariglio, N. Reyren, C. Cancellieri, and J.-M. Triscone, Phys. Rev. Lett. 104, 126803 (2010).

${ }^{13}$ S. Caprara, F. Peronaci, and M. Grilli, Phys. Rev. Lett. 109, 196401 (2012).

${ }^{14}$ C. Bell, S. Harashima, Y. Kozuka, M. Kim, B. G. Kim, Y. Hikita, and H. Y. Hwang, Phys. Rev. Lett. 103, 226802 (2009).

${ }^{15} \mathrm{~B}$. Kalisky (private communication).

${ }^{16}$ S. Ilani (private communication).

${ }^{17}$ S. Caprara, M. Grilli, L. Benfatto, and C. Castellani, Phys. Rev. B 84, 014514 (2011).

${ }^{18}$ See Supplemental Material at http://link.aps.org/supplemental/ 10.1103/PhysRevB.88.020504 for the random resistor network used to model the inhomogeneous character of the oxide interfaces and to fit the experimental resistivity curves (section A) and the two-band model used to describe superconductivity inside the superconducting puddles (section $\mathrm{B}$ ).

${ }^{19}$ Recent experiments in LTO/STO ${ }^{5}$ show evidence of inhomogeneities with typical sizes $\approx 100 \mathrm{~nm}$, thereby supporting the present assumption of similarly small puddles in LAO/STO.

${ }^{20}$ D. Stornaiuolo, S. Gariglio, N. J. G. Couto, A. Fête, A. D. Caviglia, G. Seyfarth, D. Jaccard, A. F. Morpurgo, and J.-M. Triscone, Appl. Phys. Lett. 101, 222601 (2012).

${ }^{21}$ D. Stauffer and A. Aharony, Introduction to Percolation Theory, 2nd ed. (Taylor and Francis, London, 1994).

${ }^{22}$ D. Bucheli, S. Caprara, C. Castellani, and M. Grilli, New J. Phys. 15, 023014 (2013).

${ }^{23}$ We point out that here and in Ref. 22 the fractal character (of the diffusion limited aggregation type) of the generated clusters has no intentional physical meaning, but is merely a technical artifice to generate dense, and yet filamentary, spatially correlated structures needed to reproduce tailish behavior of the the sheet resistance curves of oxide interfaces.
${ }^{24}$ We checked that bulky inhomogeneities alone (i.e., without a longdistance filamentary structure) fail to reproduce the tailish resistance (see also Supplemental Material ${ }^{18}$ ). Therefore the filamentary character with poor long-distance connectivity seems to be a mandatory feature to reproduce the low-temperature behavior of $R(T)$.

${ }^{25}$ The various parts of the resistance curves call for different and specific features of the "tree" model, so that we considered different types of filamentary structures poorly connected at long distance and rather dense at short scale. As mentioned above, the filamentary part of the model is produced by generating fractal structures of the diffusion limited aggregation or of the symmetrized random walk type (see Supplemental Material ${ }^{18}$ ). As far as the superpuddles are concerned, we systematically explored the effect of superpuddles of different size and density. We found that a smaller fraction of larger superpuddles or a larger fraction of smaller superpuddles are essentially equivalent, as long as the superpuddles do not form percolating structures (see Figs. S6-S9 in the Supplemental Material).

${ }^{26}$ D. Rakhmilevitch, I. Neder, M. Ben Shalom, A. Tsukernik, M. Karpovski, Y. Dagan, and A. Palevski, Phys. Rev. B 87, 125409 (2013).

${ }^{27}$ M. Salluzzo, J. C. Cezar, N. B. Brookes, V. Bisogni, G. M. De Luca, C. Richter, S. Thiel, J. Mannhart, M. Huijben, A. Brinkman, G. Rijnders, and G. Ghiringhelli, Phys. Rev. Lett. 102, 166804 (2009).

${ }^{28}$ Arjun Joshua, S. Pecker, J. Ruhman, E. Altman, and S. Ilani, Nature Commun. 3, 1129 (2012).

${ }^{29}$ P. Delugas, A. Filippetti, V. Fiorentini, D. I. Bilc, D. Fontaine, and P. Ghosez, Phys. Rev. Lett. 106, 166807 (2011).

${ }^{30}$ Zhicheng Zhong, Anna Töth, and Karsten Held, Phys. Rev. B 87, 161102(R) (2013).

${ }^{31}$ Detailed treatments of multiband superconductors with chemical potential near a band edge are found, e.g., in D. Innocenti, N. Poccia, A. Ricci, A. Valletta, S. Caprara, A. Perali, and A. Bianconi, Phys. Rev. B 82, 184528 (2010); D. Innocenti, S. Caprara, N. Poccia et al., Supercond. Sci. Technol. 24, 015012 (2011); A. Perali, D. Innocenti, A. Valletta, and A. Bianconi, ibid. 25, 124002 (2012).

${ }^{32}$ R. M. Fernandes, J. T. Haraldsen, P. Wölfle, and A. V. Balatsky, Phys. Rev. B 87, 014510 (2013).

${ }^{33}$ C. S. Koonce, M. L. Cohen, J. F. Schooley, W. R. Hosler, and E. R. Pfeiffer, Phys. Rev. 163, 380 (1967). 